\title{
LIBRARIES PARTNER WITH VR FOR THE SOCIAL GOOD INITIATIVE
}

Authors: Amanda Kane, Access Services Assistant II

Samuel R. Putnam, Assistant University Librarian

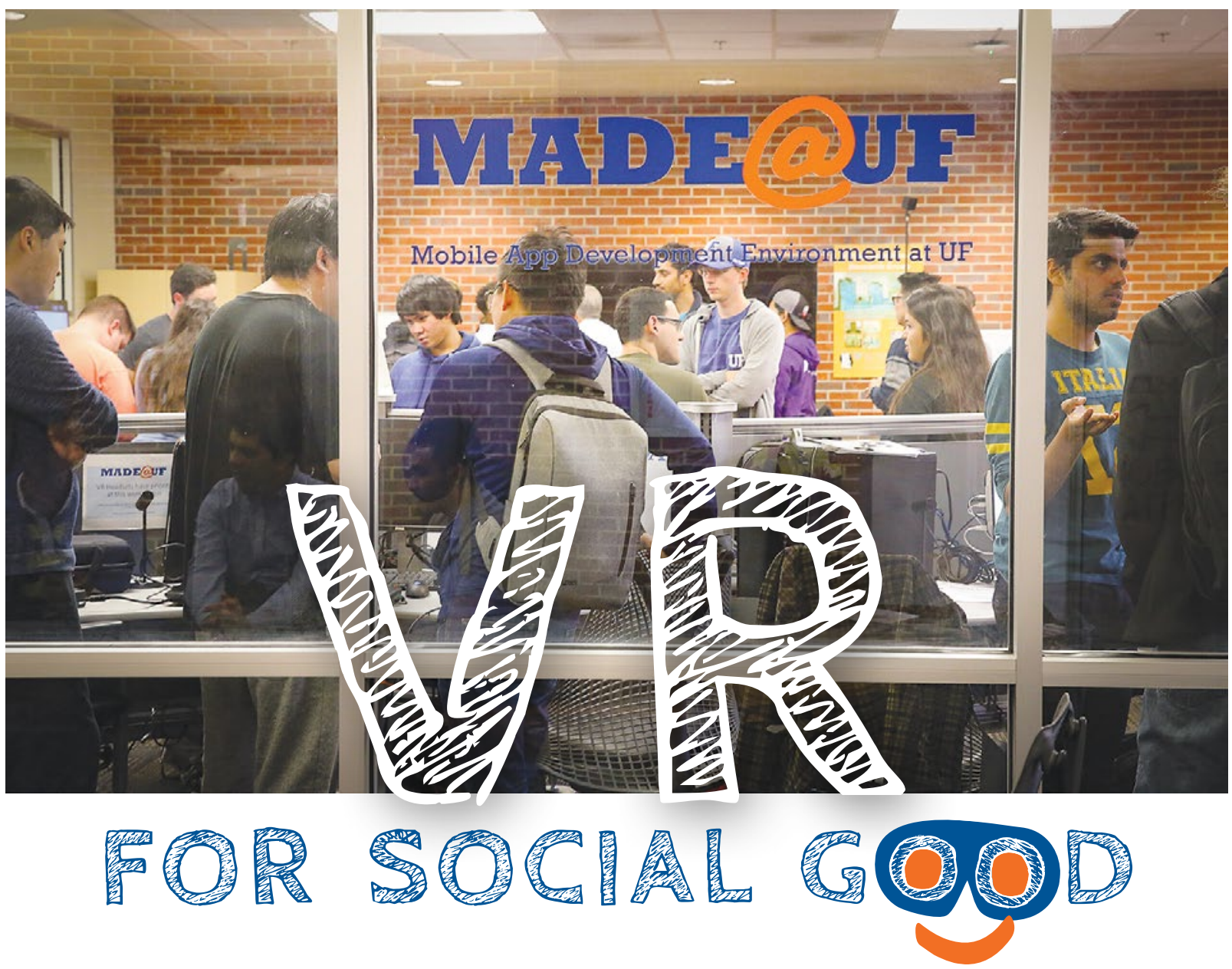

$\mathrm{n}$ an era of near constant technological advancement, expense can make it hard to fully explore the potential real-world applications of emerging technologies. This has been especially true of the fields of virtual reality (VR) and augmented reality (AR). VR's trademark head-mounted displays (HMDs) have historically been so expensive that VR has been left largely to the realm of science fiction-despite the technology having existed since the 1960s. Even as the technology has grown increasingly ubiquitous in the past several years, getting enough headsets, computers, and software to run a VR program is difficult. Marston Science Library, in partnership with the VR for the Social Good initiative, has been working to remove those barriers. 


\section{Marston Science Library's first floor is home to the MADE@UF lab, a dedicated sandbox space for developing Virtual Reality and Augmented Reality applications.}
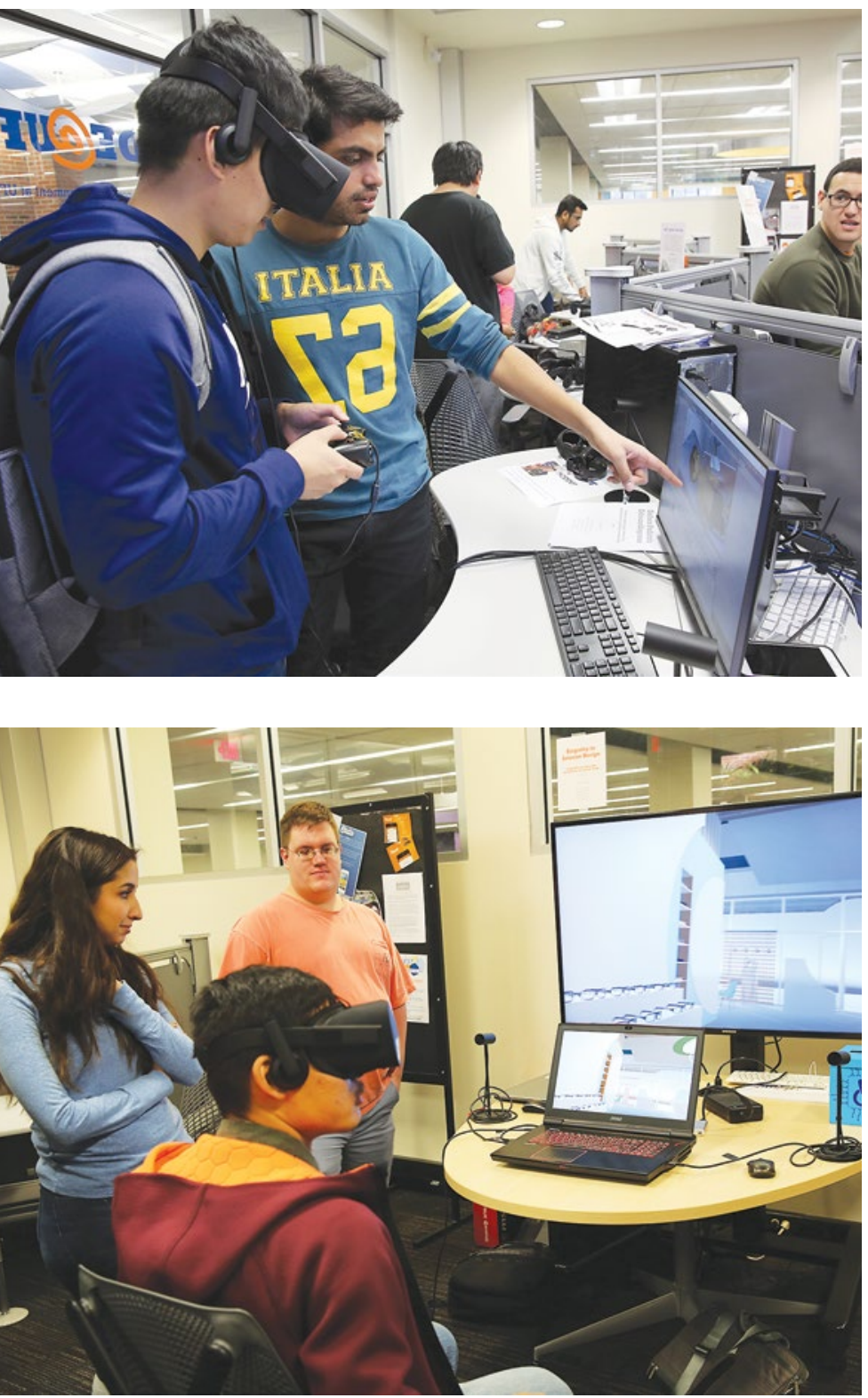

The images above show students testing $V R$ experiences created by UF students for the VR for Social Good course at VR for the Social Good Demo Day 2017.
The library loans four Oculus Rift headsets and HTC Vives for use in this space, with the necessary programs available on the MADE@UF lab computers. There are also additional HMDs that may be checked out for use outside of the library: HTC Vive, Microsoft Hololens, Google Daydream, Samsung Gear VR, and most recently, Playstation VR and Magic Leap. These VR headsets have all been purchased using money acquired from grant awards, saving library expenses and making it possible to continue expanding the VR program. This provides students with a wide variety of program types to work with, including devices they might otherwise never be able to experiment with because of the barrier of cost.

Meanwhile, the VR for the Social Good initiative connects UF students with an interest in learning about virtual and augmented reality with researchers, innovators, and entrepreneurs who believe that VR can be used as a tool to help solve social good problems. From Spring 2017 to the present, VR for the Social Good runs a course designed to teach students how to create VR experiences designed to create a positive impact on our society. Each semester, the course enrolls approximately 100 students, making it the largest enrollment VR course in the US. VR for the Social Good welcomes students from all disciplines, and required no prior experience with programming to enroll. 
Librarians at Marston Science Library will continue to provide opportunities for UF students to explore emerging VR technologies, and how they might be used to enrich the social good.

During the semesters that the course has been offered, the Marston Science Library set aside blocks of time in the MADE@UF lab that were exclusively for the use of students in the course. The students frequently borrowed library HMDs to test the programs that they were building, and were assisted with hardware and software issues by the librarian and MADE@UF director, Samuel Putnam. The Library also maintained a stock of Google Cardboard, inexpensive HMDs that work with smartphones, which were given out to the students free of charge.

At the end of each semester, the MADE@ UF lab was again reserved for the class to demo the projects they had worked on. The demos presented some truly innovative ways to use AR/VR technology to enhance the social good. One VR experience let interior design students experience their designs from the vantage point of someone in a wheelchair. Another allowed construction students to virtually tour construction sites too dangerous to visit in person. Yet another experience used a smartphone app to provide a multi-language, AR walking tour for international visitors.

In addition to the course, VR for the Social Good also supports GatorVR, a student club dedicated to learning and developing VR. GatorVR meets weekly in MADE@UF at Marston Science Library. These meetings consist of invited presentations from VR experts on campus and beyond. GatorVR also hosts workshops, open to the public, on getting started creating your own VR experience. The club also works on one collaborative VR project a semester that student members present in MADE@UF at the conclusion of each semester.

The VR for the Social Good initiative, in partnership with Marston Science Library, has barely scratched the surface when it comes to the potential good that virtual and augmented reality can offer.

\section{CHECK OUT MORE @ YOUTUBE - youtu.be/h0P4zdv8fdM}
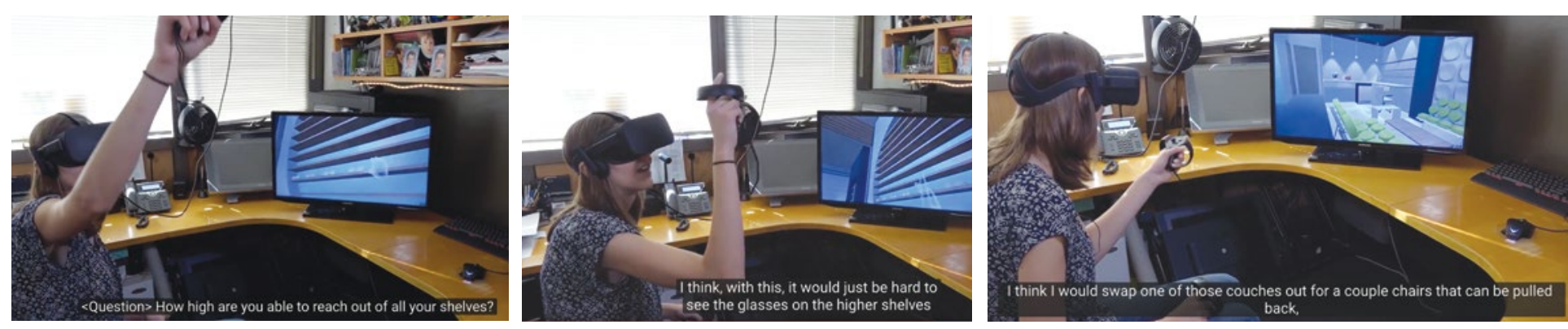

Images above from YouTube video-'Empathy in Interior Design'-using VR, design students navigate their designs from the perspective of someone who uses a wheelchair. Team: Virtually Awesome 